\title{
Prevention of the First Episode of Psychosis
}

\author{
William R. McFarlane, M.D. \\ Professor of Psychiatry, Tufts University Medical School, Director, Center for Psychiatric \\ Research, Maine Medical Center Research Institute
}

\section{Synopsis}

Long a desired goal, but increasingly a focus of research on clinical practice, prevention of psychosis has emerged as one of the most promising and impactful areas of investigational interest and effort in psychiatry. Spurred by long-term studies that have associated outcome with duration of untreated psychosis, current research is focused on improving the accuracy of prediction based on clinical and neurocognitive measures and on refining treatments for the earliest symptoms of the psychoses. Both efforts are bearing success, although there remains ambiguity as to the most effective preventive interventions. However, across five randomized clinical trials, the mean difference in rates of conversion to full psychosis is $10 \%$ to $32 \%$. This article reviews the leading studies of, and remaining issues for, this important enterprise.

\section{Keywords}

psychosis; schizophrenia; prevention; mood disorder; prodromal; high-risk

\section{Introduction}

Among all medical disorders, schizophrenia is one of the most disabling and costly. It is the most severe mental disorder, creating nearly continuous disability for a lifetime in the majority of cases. Somewhat less than one percent (between $0.27 \%$ and $0.83 \%$ ) of the population suffers from this disorder, even though the yearly incidence is low, about 0.2 per 1,000.1 In market economies, it is the fifth leading cause of disability and premature mortality among all medical disorders. It is a devastating disorder for families and communities, whose members often assume major caretaking burdens because of the profound functional deficits that this disorder imposes. It has been estimated that the total lifetime cost for a single case of schizophrenia is greater than $\$ 10,000,000.2$ Twenty-five percent $(25 \%)$ of all hospital admissions and disability payments in the U.S. are accounted by patients with severe mental illness, most of which is schizophrenia and other psychotic disorders. It is a major drain on the economy: recent estimates put direct and indirect costs to the nation for schizophrenia at more than $\$ 62$ billion per year.3 Recent meta-analyses have found that schizophrenia exacts a 25 -year reduction in life expectancy, principally secondary to heart disease, cancer and suicide. 4

Corresponding author for proofs and reprints: William R. McFarlane, M.D., Maine Medical Center, 22 Bramhall Street, Portland, ME 04102, U.S.A., (207) 662-2091, (207) 662-3300 (fax), mcfarw@mmc.org.

Publisher's Disclaimer: This is a PDF file of an unedited manuscript that has been accepted for publication. As a service to our customers we are providing this early version of the manuscript. The manuscript will undergo copyediting, typesetting, and review of the resulting proof before it is published in its final citable form. Please note that during the production process errors may be discovered which could affect the content, and all legal disclaimers that apply to the journal pertain. 
The functional disability that is particularly devastating appears to be due to biological and psychosocial deficit processes that usually begin prior to the psychotic symptoms. These often persist in spite of treatment, and usually get worse with time and each subsequent episode. While improved treatment has ameliorated some of this disability, long-term outcomes in general remain poor. Nationally, substantial reductions in the availability of community-based services have led to a decline in prognosis. At the same time, clinical trials have demonstrated that overall treatment and rehabilitation efficacy has improved substantially; thus, prognosis, in theory, should be improving. Given these realities, schizophrenia is clearly not just an interesting and clinically challenging psychiatric disorder, but also a significant public health burden.

The most significant empirical influence on the emergence of deliberate efforts to prevent psychotic disorders has been the evidence that the duration of untreated first episode of psychosis has some of the most enduring impacts on the course of illness of any known later treatment. Recent studies of first episode psychosis document that the average time between onset of psychotic symptoms and the initiation of treatment is from one to two years, depending on the study. ${ }^{5}$ These findings are troubling when one considers other studies that have shown a deteriorating course and reduced response to treatment as the duration of untreated psychosis (DUP) lengthens and with each episode of psychosis. ${ }^{6,} 7$

These and other studies suggest that the very earliest signs of development of psychosis (termed "prodromal phase") arise during adolescence as a consequence of neurodegenerative processes and psychosocial stress and hormonal changes. The current approach asserts that those processes can be altered and even reversed by earlier treatment. ${ }^{8-} 10$ In essence, present data support the conclusion that symptomatic and functional outcomes are determined by the number of days that a person has spent in a psychotic state, and perhaps by the duration of the untreated prodromal state. The prodromal period, on the other hand, can last for months to years, making its identification a feasible means of achieving the goal of indicated prevention. In addition, psychosis is inherently heterogeneous as to ultimate diagnosis. In particular, a substantial proportion of those at high risk for psychosis will actually experience the onset of a severe mood disorder, not schizophrenia. The symptomatic phenomena observed during the onset of bipolar disorder with psychosis are very similar to that for schizophrenia, with a poor prognosis. 11

Supporting prevention research is the vast amount of new knowledge that demonstrates the fundamental biological underpinnings of the disorder combined with a wealth of data showing that this biological vulnerability is heavily influenced by family, social and other environmental influences. ${ }^{12,13}$ The combined influence of these two strands of scientific and clinical progress has prompted several groups, especially in Europe and Australia, to develop treatment programs explicitly designed to intervene very early in the first episode. More recently, they have gone on to intervene in the period between onset of attenuated psychotic symptoms and the onset of frank psychosis, a period termed the prodrome, borrowing a term from epilepsy. Thus, at present, prevention of schizophrenia and other psychotic disorders constitutes secondary prevention, or in current terminology, indicated prevention. The cohort to be identified and treated preventively is, in the most technical terms, already experiencing the onset of psychotic symptoms and is often partially disabled cognitively and functionally. The extent of illness does not rise to the level needed to meet current criteria for a schizophrenic or psychotic mood disorder. For the moment, the question of whether intervention delays or prevents altogether onset is being left to longerterm research. The same is true for the etiological basis for early phase psychosis, given the current lack of a genetic marker or even clear diagnostic or prognostic implications. Even delaying onset is seen as valuable in itself, as developmental milestones and cognitive maturation appear to be defenses against the neurological consequences of long-term 
psychotic disorders. At the least, those experiencing such symptoms are vulnerable and at elevated risk for a psychotic disorder, even if they do not experience imminent onset. From a lay perspective, most persons during the prodromal phase would not be seen as mentally ill. The focus of this article is on those preventive efforts and the relevant science upon which they are based.

\section{Indicators and identifiers}

In response to the evidence for longer DUP predicting poor outcome, research has increasingly focused on the opposite tendency - to reduce DUP to the minimum possible. Most of this article is devoted to the latter alternative, but it began with this basic observation - that being psychotic for long periods leaves the patient vulnerable to increasing severity, more disability and diminished response to treatment. The practical effect was to spur development of indicators that could predict likelihood of imminent onset of psychosis. Those indicators are based on symptoms that are primarily characteristic of schizophrenia, even though psychosis itself is not limited to a single diagnostic entity. Beginning with well-known risk factors-schizoid tendencies, a family history of psychosis - the effort to develop predicting instruments to select high risk-for-psychosis subjects has centered mainly on attenuated psychotic symptoms. Thus, four well-described criteria sets have arisen, of which two are variations on the older DSM-IIIR criteria for prodromal schizophrenia. The Comprehensive Assessment for At Risk Mental States (CAARMS) 14 and the Structured Interview for the Prodromal Syndrome (SIPS), 15 define risk for psychosis under three categories: (1) attenuated psychotic symptoms, (2) brief intermittent psychotic episodes (1 week to one month), or (3) recent marked functional deterioration while having a first degree relative with a psychotic disorder or schizotypal personality disorder (SPD). The Scale for the Assessment of Basic Symptoms developed by the Bonn group and based on Huber's original concept of core or "basic" symptoms of schizophrenia, adds criteria consisting of elements of thought disorder (interference, perseveration, pressure, blockage, derealization, etc.). It achieves 78\% accuracy: nearly $70 \%$ of those meeting prodromal criteria experienced conversion to schizophrenia over 9 years. 16 Cornblatt has added a measure of negative symptoms, theorizing that almost all cases of schizophrenia will manifest subtle versions of this deficit-related syndrome even prior to onset of attenuated psychotic symptoms. 17

In a recent review of 16 follow-up studies, ${ }^{18}$ the overall average for conversion to psychosis using the various existing criteria sets is $30-35 \%$ per year. Also, serial measures in the same location, particularly Melbourne, have found a decreasing rate of conversion in untreated samples. ${ }^{19}$ That observation suggests a longer-term effect of early intervention within a specific population or a natural decrease in incidence generally. ${ }^{19}$

A frequent criticism of indicated prevention for psychosis is that those who do not convert must be false positive for psychosis, reflecting concern that ethical practice would dictate watchful waiting to determine diagnosis. 18 However, there is an emerging consensus that the great majority of such persons are often functionally and neurocognitively impaired, regardless of their near-term likelihood of developing frank psychosis. Nearly all studies of cognitive function in prodromal samples have found substantial impairment, particularly in verbal memory, processing speed, executive function and olfaction. 20 Other studies have shown lesser degrees of the same type of cortical volume reduction that has been documented in schizophrenia, especially in those who do convert. ${ }^{21}$ As well, such cases may become psychotic (true positives) later, reflecting the long risk period for onset of psychosis. ${ }^{22}$ As noted above, one study with a much longer follow-up period found a steadily increasing rate of true positives over time: $\sim 70 \% .16$ As results for indicated prevention 
improve, intervention is less and less controversial, particularly for those interventions with lower risks and general benefits for mental and physical health.

\section{Efficacy trials of indicated prevention}

The evidence for the long-term negative impact of untreated psychosis has led to early treatment being increasingly considered as the necessary step to preventing deterioration and resistance to treatment. Given the long prodromal period (often up to a year) within which it is possible to identify individuals at risk, early identification and intervention have made possible a substantial improvement in functioning, symptomatology and prognosis. Recent clinical research has shown that very early application of existing and only slightly modified evidence-based treatments for schizophrenia and mood disorders can markedly improve outcome and prognosis. Six clinical trials have consistently found that conversion to psychosis can be limited to about half or less of the expected (control) rate with medication and/or psychosocial treatment. ${ }^{23-} 28 \mathrm{We}$ turn now to the international evidence that psychosocial and/or psychopharmacological treatment may be preventive if applied prior to onset of frank psychosis (please see Table 1.).

\section{Buckingham}

Falloon described a small public health program designed to detect and treat psychotic and prodromal states as early as possible. ${ }^{29}$ It was located in Buckingham and an adjoining county in England. Treatment was continued until "all evidence of prodromal features" had remitted. Sixteen cases were identified by this system, after assessing more than one thousand referrals over the four years of the project. Thirteen cases with prodromal symptoms "experienced full and usually rapid recovery after brief integrated intervention." One case with a full psychotic onset of schizophrenia was treated to remission over four weeks; another developed bipolar disorder, also treated effectively with anti-manic medication; a third went on to experience multiple recurrences of prodromal symptoms, but not psychosis. This led to a tenfold reduction of the incidence rate in the catchment area. The integrated treatment model included family psychoeducation, thiordiazine in low doses, and follow-up via the patients' general practitioners.

\section{Manchester Cognitive Therapy Trial (EDIE)}

Morrison and his colleagues mounted a comparative clinical trial of cognitive therapy (CT) for young adults at risk of psychosis to evaluate its efficacy for the prevention of transition to psychosis. ${ }^{30}$ They randomized 58 patients at ultra-high-risk of developing a first episode of psychosis to either CT or treatment as usual. Therapy was provided over 6 months, and all patients were monitored on a monthly basis for 12 months. Logistic regression demonstrated that cognitive therapy significantly reduced the likelihood of progressing to psychosis over 12 months. In addition, it significantly reduced the likelihood of being prescribed antipsychotic medication and of meeting criteria for a DSM-IV diagnosis of a psychotic disorder and improved positive symptoms. At three years, CT significantly reduced likelihood of being prescribed antipsychotic medication, but it did not affect transition to psychosis.30 Controlling for baseline cognitive factors did yield a significant result for conversion to psychosis. However, the follow-up rate at 3 years was only $47 \%$, assessments were not blind to treatment condition, and the effect would not have been significant if two cases had not been reassigned post-hoc. As well, the mean age was 23, substantially older than samples in North America. With those caveats, cognitive therapy appears to be an acceptable and modestly efficacious intervention for the older range of those young adults whose cognitive development has been largely completed. 


\section{German Research Network on Schizophrenia}

A second trial of cognitive therapy in the prodromal phase was recently reported from the German Research Network on Schizophrenia. ${ }^{27}$ A multisite study involving centers in Cologne, Bonn, Düsseldorf and Munich, the protocol involved subjects who were in the very earliest phase, manifesting basic symptoms (e.g., thought disorder and alterations of perception) which were used, along with full psychosis, as outcome indicators. The cognitive therapy addressed thought and perception deficits, negative symptoms, general anxiety and depression and family and occupational problems. This condition was compared to supportive counseling, which comprised assessment, patient education and empathic, but unstructured, support. No medication was used. The treatment goal was to improve psychosocial and occupational functioning, which had not been shown to change in previous clinical trials in the prodromal phase. One hundred and thirteen cases were assessed, randomized and treated. Of some importance was the mean age, 25.8, which was similar to the Manchester sample in being substantially older than those in North American studies. While there were significant improvements over time for the sample in both conditions, there were no significant differences for CBT over the comparison treatment. On the other hand, deterioration did not occur and the mean level of functioning did improve in both study treatment conditions. The main methodological drawback was a large number of cases (40.7\%) with missing psychosocial outcome data, reflecting a high drop out rate. This sample was not readily comparable in that it was older and less severe in symptomatology.

\section{Danish National Schizophrenia Study of Integrated Treatment (OPUS)}

One large-scale effort that proved to be preventive in nature was originally focused on early intervention in the initial psychosis. The Danish National Schizophrenia Project (OPUS, in Danish) compared outcomes in three treatments at six sites across a prospective intervention and follow-up period of 2 years, with plans for a 5-year assessment.31 After being identified as having an initial psychosis, participants, aged 16-35, were assigned to: (1) supportive psychodynamic psychotherapy ( $\mathrm{n}=119),(2)$ integrated treatment $(\mathrm{n}=139)$ or $(3)$ treatment as usual $(n=304)$. Integrated treatment consisted of multifamily psychoeducational groups, 32 assertive community treatment and anti-psychotic medication, an approach termed FACT (Family-aided Assertive Community Treatment) in the US. It is an evidence-based approach for established schizophrenia.33 In these first episode patients, integrated treatment achieved the best results for measures of functioning and negative symptoms. Odds ratios ranged from 3 to 7 for improvements compared to treatment as usual.

A fortuitous result of the OPUS Study was that a subsample was recruited with schizotypal personality disorder (SPD) but not psychosis or schizophrenia. ${ }^{25}$ Seventy-nine such cases were assigned to the respective treatments, with the targeted outcome being initial conversion to, rather than relapse of, psychosis. At the end of two years of treatment, $48.3 \%$ of the participants in the treatment-as-usual group had a psychotic episode, compared with $25 \%$ of those in the integrated treatment arm, which was a significant difference. This study further supported the efficacy of family-based treatment as a preventive intervention, but also suggested another avenue for early identification - finding youth and young adults with SPD and offering treatment with evidence-based treatments for schizophrenia.

\section{Personal Assessment and Crisis Evaluation (PACE)}

PACE is a large-scale version of the Falloon effort. A group headed by McGorry has spent the past 12 years developing a large-scale version of the Falloon approach in Melbourne, Australia. ${ }^{23}$ The PACE Program intervened with young people who were seen to be at risk for imminent onset of psychosis. The PACE service relied on referral from the general practitioner, similarly to Falloon's project in the UK. They also targeted individuals who encounter young people frequently, including school counselors, teachers and youth 
workers. The intervention included stress management, social skills training, problemsolving, family education and crisis support, symptom monitoring and targeted medication at low doses (primarily risperidone).

The PACE program has been evaluated in an experimental design. Those who met criteria for high risk for psychosis (meeting Comprehensive Assessment for At-Risk Mental States (CAARMS) criteria) were randomly assigned to (1) a comparison group consisting of assessment and supportive treatment, an approach termed Needs-Based Intervention, or (2) an experimental group receiving cognitive therapy and symptom-specific, medication (lowdose (1-2 mg.) risperidone), given by symptom indication for six months, after which they received NBI. ${ }^{23}$ Of 522 referrals, 92 met the criteria, and of those 59 were randomly assigned and 33 refused. After 6 months, the NBI cohort had a relapse/conversion rate of $36 \%$; the experimental group receiving and adhering to intensive psychosocial treatment showed a rate of $7 \%(\mathrm{p}=0.03)$; those offered but not adhering to medication treatment manifested a rate of $12 \%$ (n.s.). Measures of functioning were unchanged from baseline. The specific-treated group had better outcomes for conversion, but over half did not accept medication, and among that group, there was a higher conversion rate during the second six month follow-up period. Interestingly, the specific- treated group, even without medication or cognitive therapy after six months, had no additional conversions. Those results held true at three years. The number needed to treat was 4 .

\section{Prevention through Risk Identification, Management and Education (PRIME)}

McGlashan and colleagues at four sites in North America used olanzapine in an industrysponsored controlled trial to treat prodromal schizophrenia and other psychotic disorders. ${ }^{24}$ Although their experience has shown that it is difficult to recruit cases using traditional drug trial methodology, the study served as a rigorous test of a pharmacological strategy to prodromal intervention. This study used an adaptation of the Australian (CAARMS) criteria, the SIPS and its associated Scale of Prodromal Symptoms (SOPS). Sixty cases were randomly assigned to olanzapine or placebo and treated for one year, followed by observation without treatment for one year. The sample averaged 17.7 years of age. The rate of conversion was 38\% for the control group and $16 \%$ for the treated group $(\mathrm{p}=.09)$, and the drop out rate was 35\% for the placebo and 55\% for the treated group. During the second year, $25 \%$ and $33 \%$, respectively, of the initially non-converting cases converted. The differences for conversion were at a trend level, but conversion in the placebo group occurred throughout the year, in contrast to the treated group, in which they occurred early in the first year. The treated group had a significantly superior effect for positive symptoms, but also experienced marked weight gain (mean $8.8 \mathrm{~kg}$.). The number needed to treat was 4.5. During the second year, without medication, symptoms worsened for both groups, but some of the weight gained was lost. A measure of functioning showed a slight, though significant, improvement over time (mean Global Assessment of Functioning (GAF) improved from 42 to 48). This study, relying almost on medication for prevention effect, found that at two years, with no treatment in the second year, $79 \%$ and $80 \%$, respectively had converted or dropped out. That was a somewhat discouraging outcome from a public health standpoint.

\section{Prevention of psychosis by Omega-3 fatty acids}

A super-nutritional strategy was tested by Amminger and colleagues, who conducted a randomized clinical trial in which omega-3 fatty acids (O-3 FAs) (without concomitant SGAs) were tested against placebo for 12 weeks, with psychosocial intervention held constant. ${ }^{28}$ At one year, the O-3 FAs condition had a conversion rate of $4.9 \%$ versus $27.5 \%$ in the placebo condition. Effects were correlated with blood level of O-3 FAs at baseline. The preventive effect persisted at one year, after the O-3 FAs were discontinued after 12 
weeks. Positive and negative symptoms and functioning all improved significantly. The number needed to treat was, again, 4 .

\section{Portland Identification and Early Referral (PIER)}

The PIER program was developed in 2000, to undertake systematic early detection, intervention and prevention of psychosis throughout an entire urban area, by providing treatment during the prodromal period. The intent was to reduce incidence of psychotic disorders and schizophrenia specifically for a defined population, while also testing the efficacy of family psychoeducation as a preventive treatment. In order to achieve effectiveness, the PIER program: (1) developed a widespread referral network, (2) mounted a major public education campaign and (3) operated a special multidisciplinary team to treat those young people at the highest risk of onset of psychosis. The initial focus of the PIER team's effort was on educating and training for all school and college counseling professionals, primary health care and pediatric physicians and mental health clinicians serving youth and young adults. The focus was on a defined catchment area of about $340,000 .{ }^{34}$ The clinical approach used was FACT, as in OPUS. Specifically, families were engaged systematically in all cases. The model was based on the fact that family intervention was highly effective in established psychotic disorders and that adolescents in particular would be difficult to engage and maintain in treatment without family involvement. 35

Preliminary outcome data are available for one year of treatment over the first six years of intake. Between 5/7/01 and 9/30/2007, of 148 prodromal participants averaging 16.5 years of age, $8.8 \%$ developed a full psychosis. The identification and referrals yielded a referral rate of $46 \%$ of the expected rate for all psychotic disorders. The mean scores for the GAF went from 38 at entry to 55 at the 12 month assessment (functioning in role as expected, but with moderate or intermittent symptoms). The PIER treatment model is currently being tested in a NIH-sponsored randomized clinical trial.

\section{Summary of Studies of Indicated Preventive Intervention}

Systems of early detection, intervention and secondary or indicated prevention have been developed and tested in the UK, Australia, Scandinavia, Germany, Austria and North America. Most involve specialized treatment with existing, empirically-validated methods. These published studies of preventive intervention have achieved a substantial reduction in rates of first frank psychosis and in reducing prodromal and psychotic symptoms. A simple compilation across studies (see Table 1) demonstrates that the mean conversion rate across studies is $9.6 \%$ of treated cases versus $32 \%$ of untreated or treatment-as-usual control cases. In three studies, the number needed to treat was 4 , comparable or superior to other medical preventive interventions.

To date, some studies have found a high drop out rate and major side effects with one medication - olanzapine. Those studies that have used evidence-based and longer-term family and rehabilitative interventions (OPUS and PIER) have had better retention rates, persistence of effects, patient social and role functioning and family wellbeing. Psychosocial functioning is emerging as the areas requiring the greatest clinical and rehabilitative effort and in which the value of psychosocial intervention is likely to be paramount. The evidence for long-term treatment effects and changes in prognosis is presently modest, much of it extrapolations of long-term outcomes of treatment for typical cases of schizophrenia. Further, some studies found that progression of disorder occurred after discontinuing a sixmonth course of treatment. However, experience demonstrates that arrest of deterioration, reduction of untreated psychosis and short-term prevention of onset of psychosis are consistent across studies and sites. The results also confirm that early detection is feasible. 
The major caveat is that no intervention strategy has yet emerged as superior to others, but rather several previously efficacious treatments for psychotic disorders have shown at least marginal efficacy in the clinical high-risk population. The most unambiguous results have followed psychosocial and super-nutritional treatment, less so after antipsychotic drug therapy.

\section{Current North American studies}

The principal barrier facing indicator and prevention intervention studies has been sample size. While schizophrenia and psychotic mood disorders have a relatively high prevalence, they have a low incidence rate. That reality makes demonstration of effects difficult at any one site, at least to the degree that would be necessary for credibility regarding policy or public health initiatives. Two studies are now underway in North America that are attempting to overcome this barrier. Both are multisite studies, and one is recruiting a large proportion of the incident at-risk population within the sites' geographic and/or service areas.

\section{Early Detection and Intervention for the Prevention of Psychosis (EDIPP) Project}

The Robert Wood Johnson Foundation (RWJF) has funded a four-year national research demonstration (EDIPP) project to produce the clinical evidence necessary to justify widespread use of the PIER early detection and preventive treatment approach. Five replication sites have been selected and are currently recruiting participants: Zucker Hillside Hospital, Glen Oaks, NY; Washtenaw County Health Organization, Ypsilanti, MI; University of California at Davis, Sacramento, CA; University of New Mexico, Albuquerque; and Mid-Valley Behavioral Health Care Organization, Salem, OR. The sites were selected to create a national sample with representatives of the major geographic, ethnic and racial subgroups within the U.S. Portland's PIER Program is the model site and the study coordinating center, while also recruiting cases. After initial training, each site has developed the three key components of the PIER model: (a) development of a community outreach and education program to train a large number of professionals from many disciplines outside the traditional mental health system; (b) targeted dissemination of information for the key affected populations regarding severe mental illness, promoting rapid consultation and treatment; and (c) a specialized FACT clinical team, whose multidisciplinary members quickly assess and treat young persons during the very earliest phases of psychosis. Each site provides family psychoeducation, supported education and employment, assertive community treatment and a psychotropic medication protocol for the prodromal population.

Each site has identified and entered into treatment $40-80$ youth, ages $12-25$, over the course of the four-year study, identified from population bases of 300-500,000. The total sample is over 340. EDIPP includes an effectiveness study with three components: (a) cut-point assignment to treatment condition, using regression discontinuity methodology (in lieu of a random assignment design); (b) an epidemiological catchment area comparison design with an historical control period and (c) a simultaneous assessment of outcomes and costeffectiveness for hospitalized (i.e., not prevented or early) first-episode patients in experimental and control catchment areas.

\section{North American Prodromal Longitudinal Study (NAPLS)}

This multisite study integrates basic measures and naturalistic outcome assessment over eight sites in the U.S. and Canada. ${ }^{36}$ Based on case selection using the same identification system as in the PIER, EDIPP, and PRIME studies (SIPS), individual sites have been coordinated to achieve a total sample of 888 cases with a high degree of agreement across 
sites on the SOPS diagnostic criteria; currently 651 cases have sufficient data for planned outcome analyses. About $10 \%$ have been recruited into medication trials, while the rest are being followed naturalistically. Most $(n=370)$ were admitted on the basis of heightened clinical risk manifested by current prodromal psychotic symptoms; the remainder consists of individuals with a first-degree relative with a psychotic illness, a DSM-IV SPD diagnosis or a recent, very brief psychotic episode. There are two comparison groups: help-seeking individuals who do not meet any of the high risk criteria $(n=174)$ and non-psychiatric controls $(n=195)$. A set of common outcome measures will be used to estimate the conversion rate, end-point diagnosis and psychosocial outcomes. The first report on an initial subsample demonstrated an overall rate of conversion of $34 \%$ by 30 months, but subgroups (e.g., those with suspiciousness, a family history and current substance abuse) converted at up to $81 \%$ of those with that clinical profile. ${ }^{37} \mathrm{~A}$ second details neurocognitive deficits compared to controls. 20

\section{Alternative interventions}

One possible treatment alternative has been suggested by a naturalistic treatment study in which psychiatrists treated subjects with psychotropic medications but without a randomized or specific drug protocol. ${ }^{38}$ Forty-eight adolescents meeting clinical high-risk criteria were treated either with SGA or serotonin reuptake inhibiting antidepressant (SSRIs) drugs. No SSRI-treated subject converted. In sharp contrast, $43 \%$ of the SGA-treated subjects converted, $92 \%$ of them after being non-adherent with their SGA medication. This study suggested that there is a major trade-off between efficacy and adherence, favoring a nonantipsychotic drug treatment approach. If confirmed in a randomized clinical trial, the SSRI approach is one that would improve the safety and reduce the negative image of a drug treatment prevention strategy. Another group, Woods and colleagues at Yale, is testing the use of glycine, a precursor of the components of the glutamine neurotransmitter system, to enhance cortical functioning in prodromal subjects.

A small body of evidence and experience is suggesting that direct practice of the neural substrate of cognition, often using sophisticated video games, may substantially improve occupational and social functioning, as well as post-treatment scores on neurocognitive testing. Currently, all results in the prodrome are preliminary but promising. The results from an earlier trial are intriguing: schizophrenia subjects experienced a larger effect on employment than on changes in the cognitive test scores. ${ }^{39}$ The more it becomes clear that positive symptoms and conversion to psychosis are preventable, the more important will it be to intervene to prevent the march of negative symptoms, cognitive deterioration and functional deficits. Those are the treatment targets that are increasingly apparent as an integral, though more subtle, and probably primary, aspect of the prodrome of psychosis.

\section{Conclusion}

As with early identification and intervention in cancer and cardiovascular disease, there are inherent challenges to indicated prevention in psychotic disorders: expanding the accuracy and evidence for the indicators, applying those indicators to earlier stages of illness and refining and testing the interventions themselves. Both a challenge and an opportunity, the next stage of development will be integrating advanced imaging techniques, neurocognitive assessments, molecular genetic strategies and knowledge of neurotransmitter functioning with improved psychosocial and psychopharmacological preventive intervention strategies to achieve enhanced accuracy and efficacy. Meanwhile, the current record of several prevention trials has raised the estimate of prevention efficacy from promising to likely, although earlier studies had substantial shortcomings. As with treatment of established schizophrenia and mood disorders, there is no currently superior single treatment modality.

Psychiatr Clin North Am. Author manuscript; available in PMC 2012 March 1. 
In fact, one interpretation of the lack of a clearly superior treatment approach is that much less intensive, risky and complex methods are effective when severity and chronicity are still minimal. This parallels approaches in cancer and heart disease. The efficacy of lowintensity/low-risk family psychoeducational intervention and O-3 FAs illustrate this point.

The complications of antipsychotic drugs argue for staged intervention approaches that begin with psychosocial therapies, such as family and cognitive methods, and supernutritional and general health and wellness methods. Antipsychotic drugs and methods, like supported employment, would be reserved for the late-stage prodrome, in which positive and negative symptoms are all but at psychotic or diagnostic levels. Given the increasing accuracy of identification methods and the evidence for the preventive efficacy of several intervention models, it appears likely that clinicians will add prevention and early intervention to their treatment approaches. The public health implications of that eventual outcome cannot be underestimated.

\section{References}

1. Eaton, WW. Evidence for universality and uniformity of schizophrenia around the world: assessment and implications. In: Gattaz, WF.; Hafner, H., editors. Search for the causes of schizophrenia. Vol. IV. Darmstadt, Germany: Steinkopf; 1999. p. 21-33.

2. Rupp A, Keith SJ. The costs of schizophrenia. Psychiatric Clinics of North America. 1993; 16(2): 413-423. [PubMed: 8332569]

3. Wu EQ, et al. The economic burden of schizophrenia in the United States. Journal of Clinical Psychiatry. 2005; 66:1122-1129. [PubMed: 16187769]

4. Parks, J.; Svendsen, D.; Singer, P.; Foti, ME. Morbidity and mortality in people with serious mental illness. Alexandria, VA: National Association of State Mental Health Program Directors, Medical Directors Council; October. 2006

5. Perkins DO, Gu H, Boteva K, Lieberman JA. Relationship between duration of untreated psychosis and outcome in first-episode schizophrenia: a critical review and meta-analysis. Am J Psychiatry. Oct; 2005 162(10):1785-1804. [PubMed: 16199825]

6. Lieberman JA, Perkins D, Belger A, et al. The early stages of schizophrenia: Speculations on pathogenesis, pathophysiology, and therapeutic approaches. Biological Psychiatry. 2001; 50(11): 884-897. [PubMed: 11743943]

7. Haas GL, Garratt LS, Sweeney JA. Delay to first antipsychotic medication in schizophrenia: Impact on symptomatology and clinical course of illness. Journal of Psychiatric Research. 1998; 32(3-4): 151-159. [PubMed: 9793868]

8. Verdoux H, Liraud F, Bergey C, Assens F, Abalan F, van Os J. Is the association between duration of untreated psychosis and outcome confounded? A two-year follow-up study of first-admitted patients. Schizophrenia Research. 2001; 49(3):231-241. [PubMed: 11356584]

9. Larsen T, More L, Vibe-Hansen L, Johannessen J. Premorbid functioning versus duration of untreated psychosis in 1 year outcome in first-episode psychosis. Schizophrenia Research. 2000; 45(1-2):1-9. [PubMed: 10978867]

10. Norman R, Townsend L, Malla A. Duration of untreated psychosis and cognitive functioning in first-episode patients. British Journal of Psychiatry. 2001; 179(4):340-345. [PubMed: 11581115]

11. Correll CU, Penzner JB, Frederickson AM, et al. Differentiation in the preonset phases of schizophrenia and mood disorders: Evidence in support of a bipolar mania prodrome. Schizophrenia Bulletin. 2007; 33(3):703-714. [PubMed: 17478437]

12. Bebbington $P$, Kuipers L. The predictive utility of expressed emotion in schizophrenia: an aggregate analysis. Psychological Medicine. 1994; 24(3):707-718. [PubMed: 7991753]

13. Tienari PA, Wynne LC, Sorri A, et al. Genotype-environment interaction in schizophreniaspectrum disorder. British Journal of Psychiatry. 2004; 184:216-222. [PubMed: 14990519]

14. Yung AR, Phillips LJ, Yuen HP, et al. Psychosis prediction: 12-month follow-up of a high-risk ("prodromal”) group. Schizophrenia Research. 2003; 60:21-32. [PubMed: 12505135] 
15. Miller TJ, McGlashan TH, Rosen JL, et al. Prodromal assessment with the Structured Interview for Prodromal syndromes and the Scale of Prodromal Symptoms: Predictive validity, inter-rater reliability and training to reliability. Schizophrenia Bulletin. 2003; 29(4):703-715. [PubMed: 14989408]

16. Klosterkotter J, Hellmich M, Steinmeyer EM, Schultze-Lutter F. Diagnosing schizophrenia in the initial prodromal phase. Archives of General Psychiatry. 2001; 58(2):158-164. [PubMed: 11177117]

17. Cornblatt B, Obuchowski M, et al. Cognitive and behavioral precursors of schizophrenia. Developmental Psychopathology. 1999; 11(3):487-508.

18. Haroun N, Dunn L, Haroun A, Cadenhead KS. Risk and protection in prodromal schizophrenia: Ethical implications for clinical practice and future research. Schizophrenia Bulletin. 2005; 32(1): 166-178. [PubMed: 16207892]

19. Yung AR, Yuen HP, Berger GE, et al. Declining transition rates in ultra high risk (prodromal) services: Dilution of reduction of risk? Schizophrenia Bulletin. 2007; 33(3):673-681. [PubMed: 17404389]

20. Seidman LJ, Giuliano AJ, Meyer EC, et al. Neuropsychology of the prodrome to psychosis in the NAPLS consortium: relationship to family history and conversion to psychosis. Archives of General Psychiatry. 2010; 67(6):578-588. [PubMed: 20530007]

21. Sun D, Phillips L, Velakoulis D, et al. Progressive brain structural changes mapped as psychosis develops in 'at risk' individuals. Schizophr Res. Mar; 2009 108(1-3):85-92. [PubMed: 19138834]

22. Kirkbride JB, Fearon P, Morgan C, et al. Heterogeneity in incidence rates of schizophrenia and other psychotic syndromes: findings from the 3-center AeSOP study. Arch Gen Psychiatry. Mar; 2006 63(3):250-258. [PubMed: 16520429]

23. McGorry P, Yung A, Phillips L, et al. Randomized controlled trial of interventions designed to reduce the risk of progression to first-episode psychosis in a clinical sample with subthreshold symptoms. Archives of General Psychiatry. 2002; 59(10):921-928. [PubMed: 12365879]

24. McGlashan TH, Zipurksy RB, Perkins D, et al. Randomized, double-blind trial of olanzapine versus placebo in patients prodromally symptomatic for psychosis. American Journal of Psychiatry. 2006; 163(5):790-799. [PubMed: 16648318]

25. Nordentoft M, Thorup A, Petersen L, et al. Transition rates from schizotypal disorder to psychotic disorder for first-contact patients included in the OPUS trial. A randomized clinical trial of integrated treatment and standard treatment. Schizophrenia Research. Jun; 2006 83(1):29-40. [PubMed: 16504481]

26. Morrison AP, French P, Walford L, et al. Cognitive therapy for the prevention of psychosis in people at ultra-high risk: Randomised controlled trial. British Journal of Psychiatry. Oct; 2004 185(4):291-297. [PubMed: 15458988]

27. Bechdolf A, Wagner M, Veith V, et al. Randomized controlled multicentre trial of cognitive behaviour therapy in the early initial prodromal state: effects on social adjustment post treatment. Early Intervention in Psychiatry. 2007; 1(1):71-78. [PubMed: 21352110]

28. Amminger GP, Schafer MR, Papageorgiou K, et al. Long-chain omega-3 fatty acids for indicated prevention of psychotic disorders: a randomized, placebo-controlled trial. Arch Gen Psychiatry. Feb; 2010 67(2):146-154. [PubMed: 20124114]

29. Falloon IRH. Early intervention for first episodes of schizophrenia: A preliminary exploration. Psychiatry. 1992; 55(1):4-15. [PubMed: 1557469]

30. Morrison AP, French P, Parker S, et al. Three-year follow-up of a randomized controlled trial of cognitive therapy for the prevention of psychosis in people at ultrahigh risk. Schizophrenia Bulletin. 2007; 33(3):682-687. [PubMed: 16973786]

31. Rosenbaum B, Valbak K, Harder S, et al. Treatment of patients with first-episode psychosis: Two year outcome data from the Danish National Schizophrenia Project. World Psychiatry. 2006; 5(2): 100-103. [PubMed: 16946951]

32. McFarlane, WR. Multifamily groups in the treatment of severe psychiatric disorders. New York, NY: Guilford Press; 2002. 
33. McFarlane W, Stastny P, Deakins S. Family-aided assertive community treatment: A comprehensive rehabilitation and intensive case management approach for persons with schizophrenic disorders. New Directions in Mental Health Services. 1992; 53:43-54.

34. McFarlane WR, Cook WL, Downing D, Verdi MB, Woodberry KA, Ruff A. Portland identification and early referral: a community-based system for identifying and treating youths at high risk of psychosis. Psychiatric Services. 2010; 61(5):512-515. [PubMed: 20439374]

35. McFarlane, WR. Family-based treatment in prodromal and first-episode psychosis. In: Miller, T., editor. Early Intervention in Psychotic Disorders. Amsterdam: Kluwer Academic Publishers; 2001. p. $197-230$.

36. Addington J, Cadenhead K, Cannon TD, et al. North American Prodrome Longitudinal Study: A collaborative multisite approach to prodromal schizophrenia research. Schizophrenia Bulletin. 2007; 33(3):665-672. [PubMed: 17255119]

37. Cannon TD, Cadenhead K, Cornblatt B, et al. Prediction of psychosis in youth at high clinical risk. Archives of General Psychiatry. 2008; 65(1):28-37. [PubMed: 18180426]

38. Cornblatt BA, Lencz T, Smith CW, et al. Can antidepressants be used to treat the schizophrenia prodrome. Results of a prospective, naturalistic treatment study of adolescents. Journal of Clinical Psychiatry. 2007; 68:546-557. [PubMed: 17474810]

39. McGurk SR, Mueser KT, Pascaris A. Cognitive training and supported employment for persons with severe mental illness: One-year results from a randomized controlled trial. Schizophrenia Bulletin. 2005; 31(4):898-909. [PubMed: 16079391] 
Table 1

Psychosis prevention studies: One year rates for conversion to psychosis

\begin{tabular}{lcccc}
\hline & \multicolumn{2}{c}{ Control } & \multicolumn{2}{c}{ Experimental } \\
& $\mathbf{n}$ & Converted & $\mathbf{n}$ & Converted \\
PACE & 28 & $10(35.7 \%)$ & 31 & $6(19.4 \%)$ \\
PRIME & 29 & $11(37.9 \%)$ & 31 & $5(16.1 \%)$ \\
OPUS & 30 & $10(33.3 \%)$ & 37 & $3(5.7 \%)$ \\
EDIE & 23 & $6(26.1 \%)$ & 35 & $2(5.7 \%)$ \\
O-3 FAs & 40 & $11(27.5 \%)$ & 41 & $2(4.9 \%)$ \\
PIER & 0 & 0 & 148 & $13(8.8 \%)$ \\
Total & 150 & 48 & 323 & 31 \\
Mean conversion rate & & $\mathbf{3 2 . 0 \%}$ & & $\mathbf{9 . 6 \%}$ \\
Without PIER & & & & \\
Total & 150 & 48 & 175 & 18 \\
Mean conversion rate & & $\mathbf{3 2 . 0 \%}$ & & $\mathbf{1 0 . 3 \%}$ \\
\hline
\end{tabular}

Psychiatr Clin North Am. Author manuscript; available in PMC 2012 March 1. 